\title{
Application Research on Testing Efficiency of Main Drainage Pump in Coal Mine Using Thermodynamic Theories
}

\author{
Deyong Shang \\ School of Mechanical Electronic \& Information Engineering, China University of Mining and Technology (Beijing), Beijing, China \\ Correspondence should be addressed to Deyong Shang; shangdy1983@126.com
}

Received 23 September 2016; Revised 15 November 2016; Accepted 29 November 2016; Published 5 January 2017

Academic Editor: Tariq Iqbal

Copyright (C) 2017 Deyong Shang. This is an open access article distributed under the Creative Commons Attribution License, which permits unrestricted use, distribution, and reproduction in any medium, provided the original work is properly cited.

\begin{abstract}
The efficiency of a drainage pump should be tested at regular intervals to master the status of the drainage pump in real time and thus achieve the goal of saving energy. The ultrasonic flowmeter method is traditionally used to measure the flow of the pump. But there are some defects in this kind of method of underground coal mine. This paper first introduces the principle of testing the main drainage pump efficiency in coal mine using thermodynamic theories, then analyzes the energy transformation during the process of draining water, and finally derives the calculation formulae of the pump efficiency, which meet the on-site precision of engineering. On the basis of analyzing the theories, the protective sleeve and the base of the temperature sensor are designed to measure the water temperature at inlet and outlet of the pump. The efficiencies of pumps with two specifications are measured, respectively, by using the thermodynamic method and ultrasonic flowmeter method. By contrast, the results show that thermodynamic method can satisfy the precision of the testing requirements accuracy for high-flow and high-lift drainage pump under normal temperatures. Moreover, some measures are summed up to improve the accuracy of testing the pump efficiency, which are of guiding significance for on-site testing of the main drainage pump efficiency in coal mine.
\end{abstract}

\section{Introduction}

The main drainage pump system is one of the major energy consumption equipment tools in coal mine. The running state of the pump is important to the security of the coal mine [1]. According to the requirements of Safety Regulations in Coal Mine in china, in order to ensure the drainage pump economic and efficient operation, it is necessary to test the performance of the pump at regular intervals to make the pump work in the high efficiency area, which is closely related to mining security and efficiency.

At present, the traditional testing process for the pump efficiency is to test the parameters of each working condition, such as the pressure gap between the inlet and outlet, instantaneous flow, motor voltage and electric current, and revolving speed and then calculate the parameters such as the pump efficiency and the power consumption per ton of water in water supply system by the traditional formula [2]. This method needs to measure the flow of the pump accurately, but it is hard to measure accurately due to the restrictions in some special circumstances, for example, in pumping house underground coal mine. The ultrasonic measurement method is the most commonly used method to measure the pump flow which is accurate and easy to operate. But, due to space limitations, it is difficult to install the ultrasonic flowmeter in the right place in pumping house underground of the coal mine. Due to the chemical properties of coal mine water, a lot of coal mine drainage pipe scaling's phenomenon is very serious [3], weakening the signal intensity of ultrasonic flowmeter, resulting in test flow failure. Also there will be measuring errors using the ultrasonic flowmeter due to the entrained air and bubble in pipes and the influence to the ultrasonic flowmeter caused by protective paint of the outside of drain-pipes and the high strength electromagnetic interference by high power electrical machinery, and so forth. Therefore, it is urgent to develop a method for testing the pump efficiency, which is simple and easy to execute and convenient for on-site testing at regular intervals underground of coal mine. Based on the above reasons, this paper presents the method of using temperature difference to test the main water pump efficiency in coal mine. 


\section{Brief Introduction of Thermodynamics Method}

The thermodynamics method (micro temperature differential method) for testing the pump efficiency was firstly applied in France in 1954 and then adopted by the UK, Germany, and so forth one after another and now has been brought into the International Electric Committee Standard (IEC 60778). In December 1980, this method was put forward formally in The Research Report about Water Pump Automation and Energy Saving by Bai Mingsheng. Although this method is simplified according to different measure environments, it can also guarantee a high precision if some steps are taken during the efficiency test. Therefore, the test results of this method are reliable and can meet the accuracy requirements of test in engineering site [4-7].

The thermodynamics method can calculate pump efficiency by measuring the thermodynamic parameter (temperature, the pressure of the inlet and outlet, etc.) and the physical properties (density, specific heat, etc.) of water according to thermodynamic law [8]. The friction between water and the interior of the case of pump comes about when the water flows through the pump. Meanwhile, the friction, turbulence, and lash also come about in the inner of water flow, which results in loss of the mechanical energy of water flow. The water power phenomenon of the inner of the centrifugal pump is complicated, and the main energy losses include mechanical loss, volume loss, and hydraulic loss. According to the conservation law of energy and the transformation law of energy, the loss of mechanical energy will certainly transform into thermal energy entirely without considering the heat exchange between the water flow and the outside and the loss of sound power; thus the temperature of the water flow at the outlet of the pump rises. In other words, the difference in temperature between the inlet and outlet of the pump comes about, and specific enthalpy of the water flow changes. We can calculate the hydraulic pump efficiency through measuring the specific enthalpy change.

\section{Formulae of Thermodynamics Applying to On-Site Measurement}

According to the Bernoulli equation and the similarity theory of pump operation, we can assume the drainage pump as an opening system in order to analyze the energy transformation during the process of draining. The process is shown in Figure 1.

$$
\begin{aligned}
& m\left(h_{2}+\frac{v_{2}^{2}}{2}+g z_{2}\right) \times 10^{-3} \\
& \quad=m\left(h_{1}+\frac{v_{1}^{2}}{2}+g z_{1}\right) \times 10^{-3}+N_{z}-N_{s},
\end{aligned}
$$

where $m$ is mass flow $(\mathrm{kg} / \mathrm{s}), h_{1}, h_{2}$ are enthalpy of water of unit mass $(\mathrm{J} / \mathrm{kg}), v_{1}, v_{2}$ are velocity of flow $(\mathrm{m} / \mathrm{s}), z_{1}, z_{2}$ are center data mark of the cross section of measurement $(\mathrm{m})$, $N_{z}$ is input power of pump shaft $(\mathrm{kW}), N_{s}$ is stray loss power $(\mathrm{kW})$, and $g=9.81 \mathrm{~m} / \mathrm{s}^{2}$.

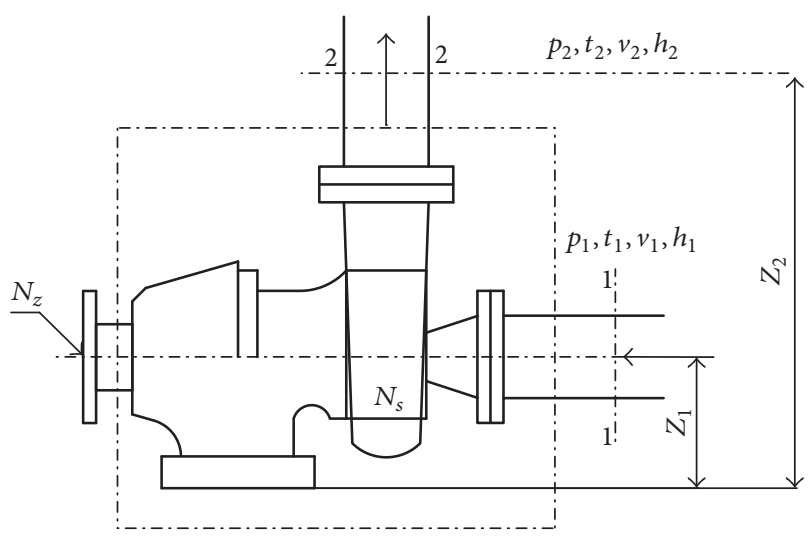

Figure 1: Pump's energy conversion.

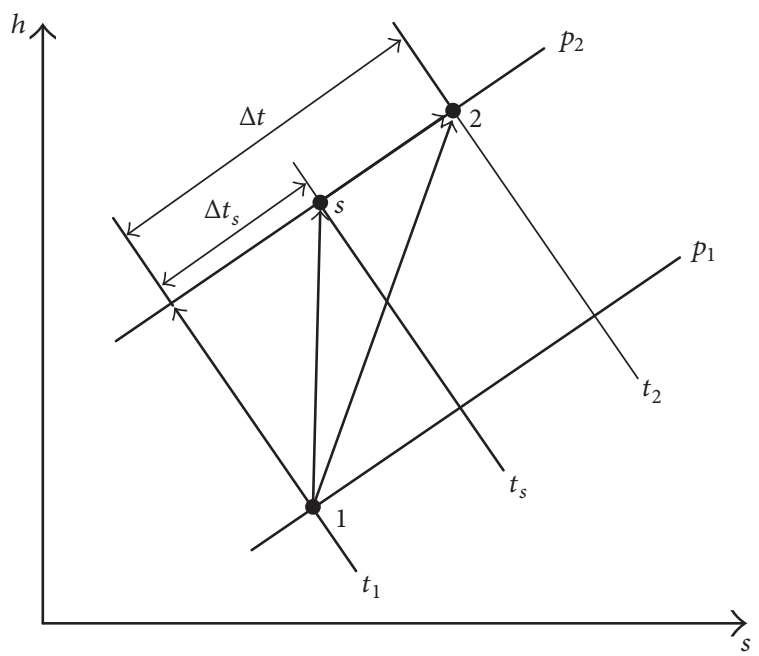

FIGURE 2: The enthalpy-entropy diagram of pump.

The stray loss power $N_{s}$ of the pump shaft includes three parts: the radiating loss $N_{r}$ of the outside of the pump body, mechanical loss $N_{m}$ by friction between the pump shaft and the bearing, filling, shaft end seal, and leakage flow loss $N_{x}$ of the balancing device and the shaft seal device. $N_{s} / N_{z}$ is small and taken as $0.01-0.03$ according to different conditions during the process of on-site test [9].

The enthalpy and entropy change of the water during the thermal process of the pump will be analyzed hereinafter. The process is shown in Figure 2.

Enthalpy is a status parameter, and the enthalpy difference only rests with the thermodynamic parameter and is irrelevant to process. Thus, the real process of water flowing through the pump $1 \rightarrow 2$ can change forms at will. Entropy remains unchanged as water flows from state point 1 $\left(h_{1}, t_{1}, p_{1}, z_{1}, v_{1}\right)$ of the pump inlet to state $S\left(h_{s}, t_{s}, p_{2}, z_{2}, v_{2}\right)$ and then along the constant-pressure line $p_{2}$ to make the loss of the pump change into the thermal energy by heating and finally flowing to state point $2\left(h_{2}, t_{2}, p_{2}, z_{2}, v_{2}\right)$ of the pump 
outlet. In other words, this process is an isentropic process. The liquid enthalpy difference can be expressed as

$$
h_{2}-h_{1}=\left(h_{2}-h_{s}\right)+\left(h_{s}-h_{1}\right) \text {. }
$$

By the thermodynamic relation equations " $d h=T d s+$ $v d p$ " and " $d s=0$ " in the isentropic process, it can be obtained that

$$
h_{s}-h_{1}=\int_{1}^{s} v d p=\bar{v}\left(p_{2}-p_{1}\right)=\frac{p_{2}-p_{1}}{\bar{\rho}} \text {, }
$$

where $\bar{v}$ is the average specific volume of water $\left(\mathrm{m}^{3} / \mathrm{kg}\right)$ and $\bar{\rho}$ is the average density of water $\left(\mathrm{kg} / \mathrm{m}^{3}\right)$.

It can be obtained that

$$
\begin{aligned}
d h & =T d s+v d p=T\left[\frac{c_{p}}{T} d T-\left(\frac{\partial u}{\partial T}\right)_{p} d p\right]+v d p \\
& =c_{p} d T+\left[v-T\left(\frac{\partial u}{\partial T}\right)_{p}\right] d p .
\end{aligned}
$$

In the isobaric process, $d p=0$, the enthalpy difference can be obtained from (4) as

$$
h_{2}-h_{s}=\int_{s}^{2} c_{p} d T=\overline{c_{p}}\left(t_{2}-t_{s}\right),
$$

where if $c_{p}$ has not changed much in the investigative range, it can be replaced with the average value $\overline{c_{p}}$ which is an average liquid isobaric specific heat capacity in the range of the pressure $P_{2}$ and the temperature is $t_{2} \sim t_{s}$.

$$
t_{2}-t_{s}=\left(t_{2}-t_{1}\right)+\left(t_{1}-t_{s}\right)=\Delta t-\Delta t_{s},
$$

where $\Delta t_{s}$ is the isentropic temperature rise of water, which can be found from the matter-properties data of water [10].

\section{Equation of Pump Efficiency Satisfying the Precision of Engineering Test}

According to the standard AQ 1012-2005 of The Main Drainage System Safety Testing Inspection Specifications in Coal Mine [11], the $\mathrm{C}$ level precision as required by the standards is sufficient for the performance test of the pump in daily testing. Based on the standard GB3216-89, The Centrifugal Pump, the Mixed Flow Pump, Axial Flow Pump, and Vortex Pump Test Method in China [12], the C level precision of the pump efficiency defined as the maximum possible differences between the test performance and actual performance is $5 \%$. So the items, energy losses of which account for small fractions of the total energy during the process of energy exchange, can be ignored or replaced with the corrected value.

Water flows from Section 1 of the pump inlet to $S$ point along the line of isentropic change, and the power consumed during this process is seen as effective power and is hydraulic energy acquired after water flowing through the pump case, the computation formula of which is shown in

$$
\begin{aligned}
N_{e}= & m\left[\left(h_{s}-h_{1}\right)+\frac{\left(v_{2}^{2}-v_{1}^{2}\right)}{2}+g\left(z_{2}-z_{1}\right)\right] \\
& \times 10^{-3} \\
= & m\left[\left(\frac{p_{2}-p_{1}}{\bar{\rho}}\right)+\frac{\left(v_{2}^{2}-v_{1}^{2}\right)}{2}+g\left(z_{2}-z_{1}\right)\right] \\
& \times 10^{-3}=m g H \times 10^{-3},
\end{aligned}
$$

where $H$ is lift of pump (m).

The computation formula of shaft power can be defined as

$$
\begin{aligned}
N_{z} & =m\left[\left(h_{2}-h_{1}\right)+\frac{v_{2}^{2}-v_{1}^{2}}{2}+g\left(z_{2}-z_{1}\right)\right] \times 10^{-3} \\
& +N_{s}=m\left[\left(\frac{p_{2}-p_{1}}{\bar{\rho}}\right)+\frac{\left(v_{2}^{2}-v_{1}^{2}\right)}{2}+g\left(z_{2}-z_{1}\right)\right. \\
& \left.+\overline{c_{p}}\left(t_{2}-t_{s}\right)\right] \times 10^{-3}+N_{s} .
\end{aligned}
$$

The computation formula of the pump efficiency is shown (9) based on the definition of the pump efficiency.

$$
\begin{aligned}
\eta & =\frac{N_{e}}{N_{z}}=\frac{m\left[\left(\left(p_{2}-p_{1}\right) / \bar{\rho}\right)+\left(v_{2}^{2}-v_{1}^{2}\right) / 2+g\left(z_{2}-z_{1}\right)\right] \times 10^{-3}}{m\left[\left(\left(p_{2}-p_{1}\right) / \bar{\rho}\right)+\left(v_{2}^{2}-v_{1}^{2}\right) / 2+g\left(z_{2}-z_{1}\right)+\overline{c_{p}}\left(t_{2}-t_{s}\right)\right] \times 10^{-3}+N_{s}} \\
& =\frac{m g H}{m g H+m \overline{c_{p}}\left(\Delta t-\Delta t_{s}\right)+N_{s} / N_{e}}=\frac{1}{1.02+\overline{c_{p}}\left(\Delta t-\Delta t_{s}\right) / g H} .
\end{aligned}
$$

During testing of the pump efficiency under normal temperature, $N_{s} / N_{e}$ can be chosen as 0.02 [9]. In this paper. It can be seen from (9) that the pump efficiency is relevant to the constant-pressure specific heat capacity of water $\left(c_{p}\right)$, the difference in temperature at inlet and outlet of the pump $(\Delta t)$, the isentropic temperature rise of water
$\left(\Delta t_{s}\right)$, the acceleration of gravity $(g)$, and the pump head $(H)$.

During testing of the pump of normal temperature, the specific heat of water has not changed very much and can be chosen as $c_{p}=4186.8 \mathrm{~J} /(\mathrm{kg} \cdot \mathrm{k})$. At the same time, the isentropic temperature rise $\Delta t_{s}$ can be ignored [13]. 
The equation of head of delivery is

$$
H=\left(\frac{p_{2}-p_{1}}{\bar{\rho}}\right)+\frac{\left(v_{2}^{2}-v_{1}^{2}\right)}{2}+g\left(z_{2}-z_{1}\right) \text {. }
$$

For the pump whose inlet diameter and outlet diameter are equal in coal mine, by choosing $v_{2}=v_{1}$, then (9) can be changed into the following equation:

$$
\begin{aligned}
\eta & =\frac{N_{e}}{N_{z}} \\
& =\frac{1}{1.02+426.789 \Delta t /\left(\left(\left(p_{2}-p_{1}\right) / \bar{\rho}\right)+g\left(z_{2}-z_{1}\right)\right)} .
\end{aligned}
$$

The constant-pressure specific heat capacity of water $c_{p}$, the density of water $\rho$, and the acceleration of gravity $g$ are all constants. It can be seen from (11) that if you want to test the pump efficiency, you only need to measure the temperature difference at the inlet and outlet of the pump $\left(\Delta t=t_{2}-t_{1}\right)$, the pressure difference $\left(\Delta p=p_{2}-p_{1}\right)$, and the horizontal elevation difference $\left(\Delta z=z_{2}-z_{1}\right)$. This is just a pump efficiency test of thermodynamics method.

It is a new way to test the efficiency of the main drainage pump in coal mine by a thermodynamics method, by which less parameters are tested and the test results can be obtained easily, and it is unaffected by the inaccurate results of flow measurement. In a word, this method is simple and suitable for testing the efficiency of the main drainage pump underground of the coal mine.

\section{On-Site Testing Scheme Design of the Drainage Pump}

It can be seen from the thermodynamics derivation equation (11) of pump efficiency that the temperature difference at the inlet and outlet of the pump, the pressure difference, and the horizontal elevation difference are main parameters for testing the pump efficiency. After testing the efficiencies of the pump and then the power and the speed of the input shaft of the pump in every operating mode, the performance parameters, such as the flow of the pump and the power consumption per ton of water, can be calculated by a traditional calculating equation of pump efficiency.

\subsection{Testing Scheme of the Temperature of Water Flowing} through a Drain-Pipe. It is necessary to accurately test the temperature of water, which has much influence over the calculating results of pump efficiency. It is better to choose a temperature sensor with the precision of $0.001-0.020^{\circ} \mathrm{C}$, and if the site where the temperature of water is tested is far away from the inlet and outlet of the pump, a resistance-type thermometer of four-wire system in order to design a proper bridge should be chosen to eliminate the electric resistance connecting wires for raising the testing precision as much as possible. In this paper, a temperature sensor (PT100) of fourwire system with the precision of $\pm 0.005^{\circ} \mathrm{C}$ is used, as shown in Figure 3.

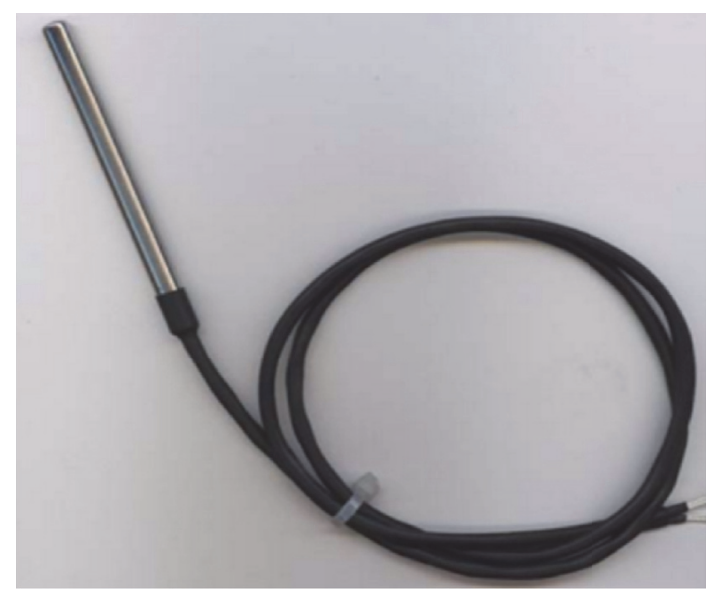

FIGURE 3: Four-wire PT100 temperature sensor.

In order to test the temperature of water at inlet and outlet of the pump conveniently and accurately, the protective sleeve over the temperature sensor as shown in Figure 4 is designed, the length of which is machined based on different diameters of the drain-pipes.

Insert the temperature sensor into the protective sleeve during testing, and make the sensor tight against the inner wall and bottom of the sleeve. Inject the water in a water sump into a sleeve until the thermometric probe is immersed to make the temperature of water in the sleeve close to the one in the pump in order to conduct heat uniformly. The installation site of the sleeve is shown as Figure 5.

It is required to drill at the water inlet and the drainpipe of the main drainage pump in order to install the sleeve. The base for the protective sleeve and the matching spiral trunk piston are designed as shown in Figure 6. The bottom of the base designed is an incline and welded at the lower part of the sluice value of the drain-pipe and the water inlet shown in Figure 5 in order to keep the water of the sleeve from flowing out, because the drain-pipe is vertical. When the testing is finished, get the temperature sensor out of the protective sleeve and drill with the spiral trunk piston as shown in Figure 6 hermetically for next test.

\subsection{Testing Scheme for Pressure at the Inlet and Outlet of} the Pump. It is necessary that the main drainage pump is equipped with a vacuum gauge and a delivery gauge under "Safety Regulations in Coal Mine." When testing the pressure at the inlet and the outlet of the pump, it is only necessary to remove the two gauges and then install pressure sensors as shown in Figure 7. The sensors with different ranges are selected in order to improve the testing precision and installed, respectively, at the sites where the gauges were. It is important to note that the sensor with smaller range should be installed at the location where the vacuum gauge is and the sensor with larger range at the location where the delivery gauge is, the exchange being prohibited. During the process of wiring and testing, keep water from the connector assembly of the pressure sensor; otherwise, the testing will not be done normally. 

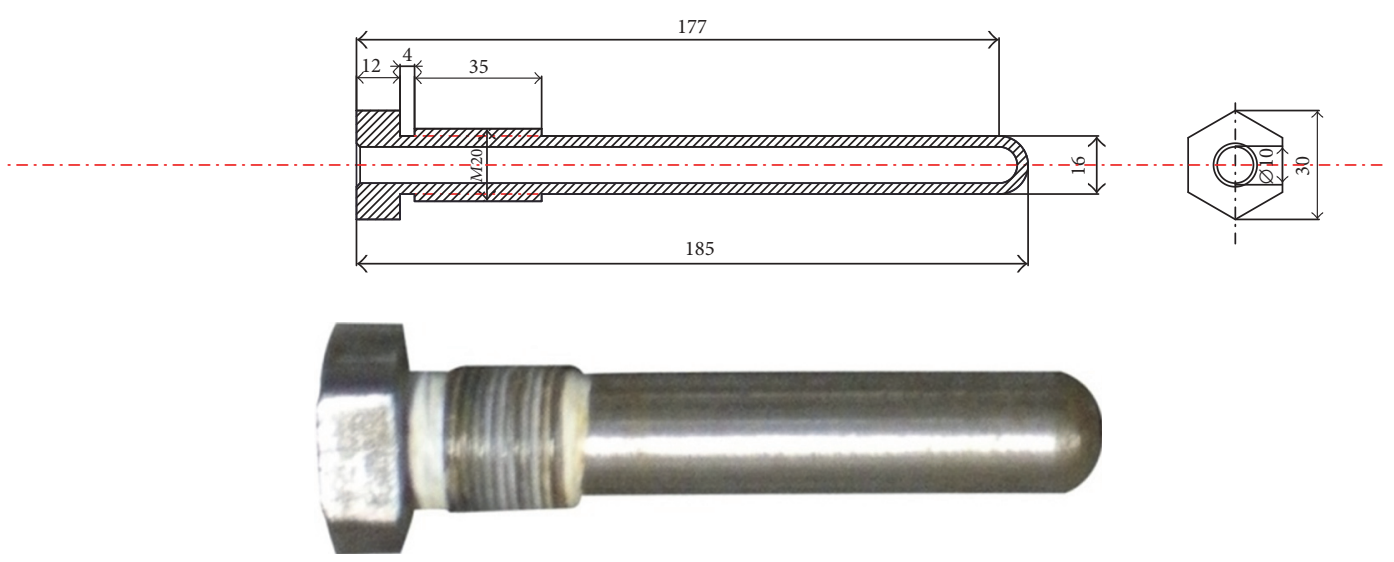

FIGURE 4: Schematic view of protective sleeve.

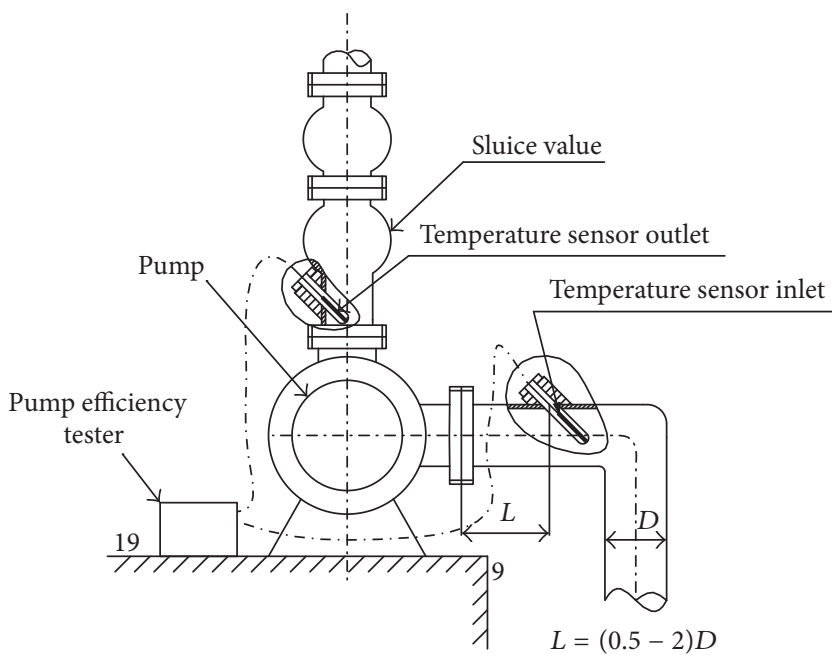

FIGURE 5: Installation view of the sleeve.

5.3. Testing Schemes of Other Parameters. This paper adopts a photoelectric revolution-speed sensor to collect revolutionspeed pulse signal due to limitation by on-site environment underground coal mine, which is a noncontact measurement. Use the testing device operated economically by an electrical machinery to test the power of input pump shaft and the electrical parameters of the electrical machinery, such as the voltage, current, active power, and power factor. The data from every sensor is linked with an upper monitor by a data acquisition card and a signal processing card, thereby realizing automatic online monitoring.

5.4. Implementation Process of Testing. Both the temperature difference and the pressure difference are main parameters which have a strong influence on the test of pump efficiency. In order to ensure precision of testing, it is necessary to calibrate both the temperature sensors and the pressure sensors at inlet and outlet first before installing. Put two temperature sensors into a water sump and calibrate them when they are stable. The pressure sensors may be placed at the same position in a pump house to be calibrated.
Then start the pump to test after confirming that all sensors have been installed accurately. The sensors at water inlet are installed as shown in Figure 8.

It is necessary to make the pump operate over twenty minutes before collecting the data. During the whole testing process, the working conditions of the pump can be adjusted through changing the size of the opening of a plate gate valve in the drainage pipeline from the biggest opening to shut-off, particularly getting 5-7 points of working condition uniformly. It is better to change the size of the opening uniformly in order to make the working conditions of the pump cover the whole working area, fully disclosing the operating performance of the main drainage pump. The pump should be operated over twenty minutes at the working condition after each adjustment, and the data should be collected when the pump is stable in order to ensure the accuracy of testing.

\section{Analysis of the Testing Results}

The efficiency of a main drainage pump of MD450-60 ×6 in a coal mine is tested through the thermodynamic method and the ultrasonic flowmeter method simultaneously, the data of pump being as follows: rated flow of the pump is $450 \mathrm{~m}^{3} / \mathrm{h}$, the rated head is $360 \mathrm{~m}$, the rated efficiency is $78 \%$, the rated speed is $1480 \mathrm{r} / \mathrm{min}$, the type of mating electric machine is JS158-4, the rated power is $680 \mathrm{~kW}$, the stator voltage is $6000 \mathrm{~V}$, the stator current is $79 \mathrm{~A}$, the rated speed is $1485 \mathrm{r} / \mathrm{min}$, the rated efficiency is $94 \%$, the internal diameter of drain line is $0.35 \mathrm{~m}$, actual drainage height is $304 \mathrm{~m}$, the vertical distance of pressure gauges is $0.45 \mathrm{~m}$, and the suction height is $3.5 \mathrm{~m}$. The data of testing using the thermodynamic method are shown in Table 1, and the data of testing using the thermodynamic method are shown in Table 2.

The graph of pump efficiency transformed into rated condition as shown in Figure 9; we can see that the measurement of the pump efficiency curve (black solid line Q- $\eta$ in Figure 9) tested by the thermodynamic method is rather similar to the measurement result curve by the ultrasonic flowmeter method (the red dashed line Q- $\eta$ in Figure 9), and the test precision by the thermodynamic method is closer to 


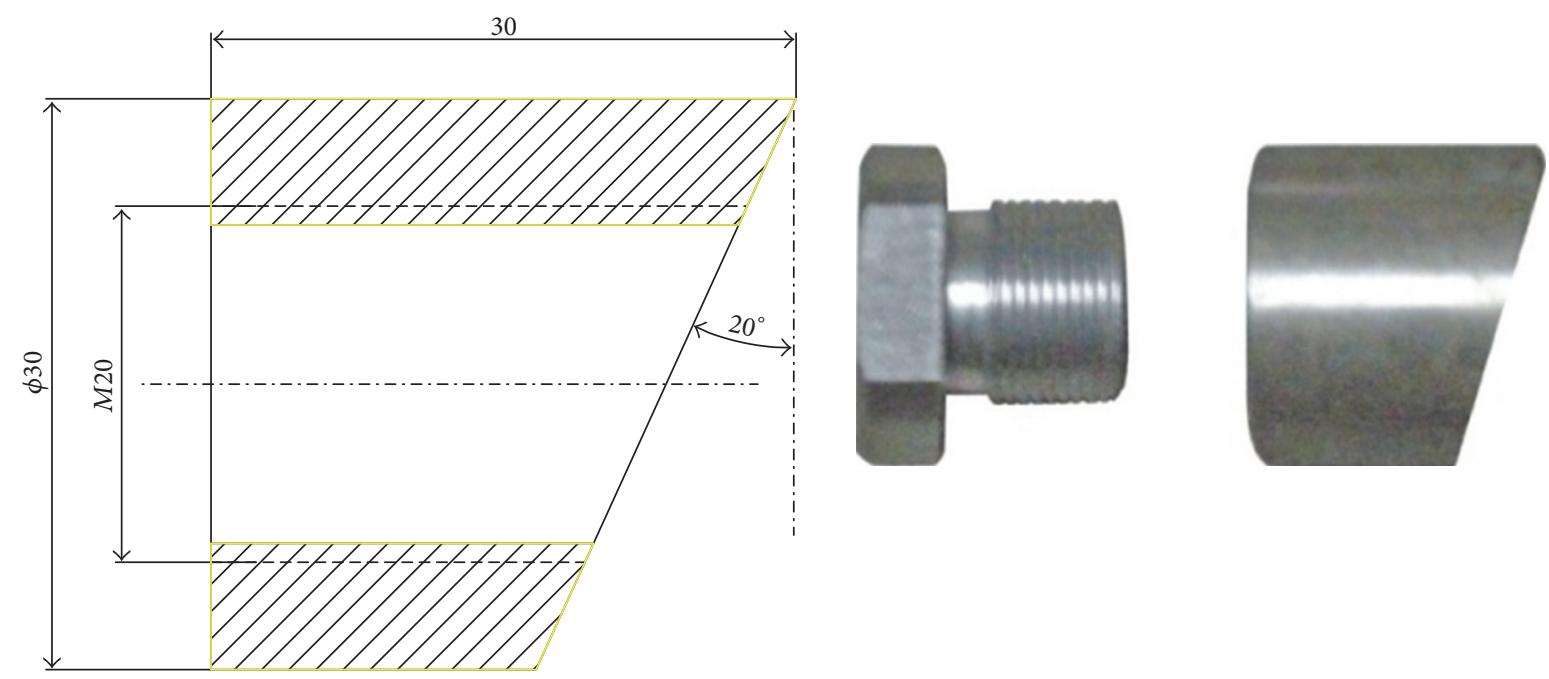

Figure 6: The base.

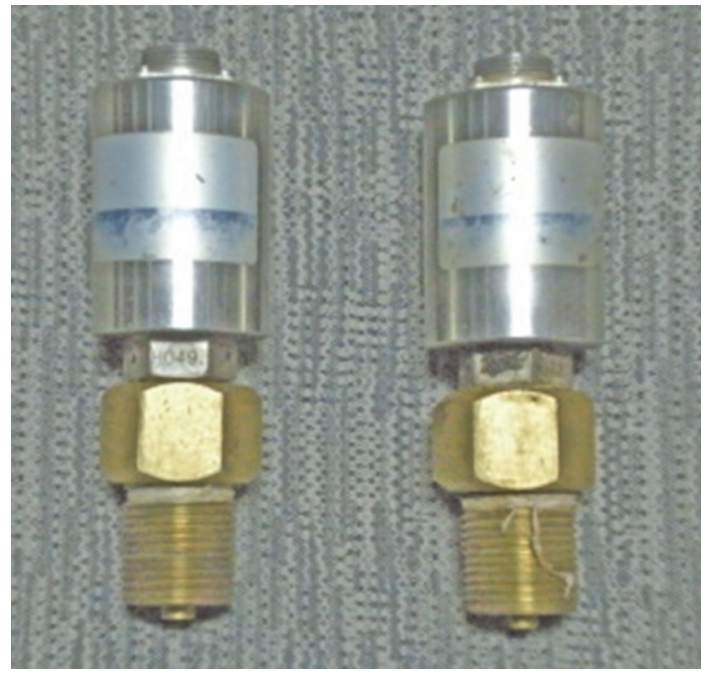

FIgURE 7: Pressure sensor.

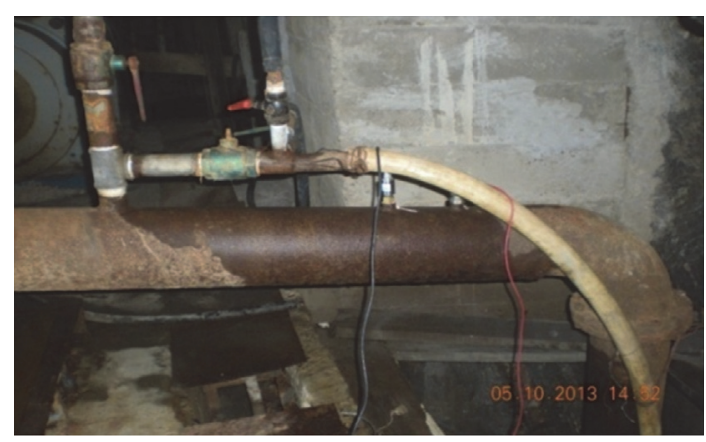

FIGURE 8: Sensors installation instruction in water inlet.

the precision by the ultrasonic flowmeter method with the increase of the pump flow. By comparison, the test results data are close to the ultrasonic flowmeter method, and the

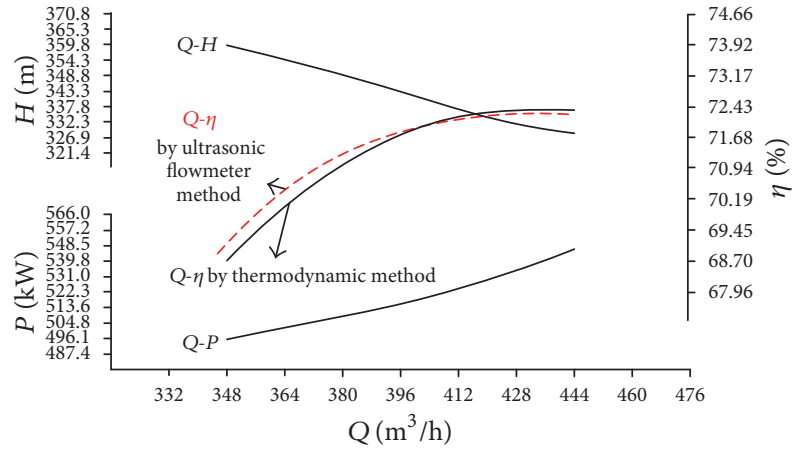

FIgURe 9: Performance graph of the pump.

thermodynamic method being used in coal mine to test the pump efficiency is feasible.

In addition, the drainage pump of D46-30 $\times 5$ is tested through a thermodynamic method, and the data of pump are as follows: rated flow of the pump is $46 \mathrm{~m}^{3} / \mathrm{h}$, the rated head is $150 \mathrm{~m}$, the rated efficiency is $72 \%$, the rated speed is $2950 \mathrm{r} / \mathrm{min}$, the type of mating electric machine is Y200-2, the rated power is $37 \mathrm{~kW}$, and the stator voltage is $380 \mathrm{~V}$. During the testing process, it is found that this type of pump has small flow and low head of delivery that the loss of mechanical energy is very small (in conjunction with other reasons, e.g., heat radiation), which will not be transformed into enough thermal energy of water flow and makes it uneasy to collect the temperature parameters, since the changes of temperature parameters are small under every working condition.

It is known from the on-site experiment that the thermodynamics method for testing the pump efficiency is applied to the pump with large flow and high head of delivery and not the small-sized pump because the temperature change at outlet thereof is so small that the pump efficiency cannot be measured accurately. 
TABLE 1: The test data through the thermodynamic method.

\begin{tabular}{|c|c|c|c|c|c|c|c|}
\hline \multirow{2}{*}{ Number } & \multirow{2}{*}{ Item } & \multirow{2}{*}{ Unit } & \multicolumn{5}{|c|}{ The adjustment point of flow } \\
\hline & & & 1 & 2 & 3 & 4 & 5 \\
\hline$\overline{(1)}$ & The temperature at inlet & ${ }^{\circ} \mathrm{C}$ & 18.561 & 18.563 & 18.563 & 18.560 & 18.561 \\
\hline$(2)$ & The temperature at outlet & ${ }^{\circ} \mathrm{C}$ & 18.835 & 18.911 & 18.955 & 19.205 & 19.426 \\
\hline$(2)$ & The reading of vacuum gauge & $\mathrm{MPa}$ & 0.034 & 0.036 & 0.037 & 0.039 & 0.040 \\
\hline (3) & The reading of pressure gauge & $\mathrm{MPa}$ & 3.207 & 3.293 & 3.381 & 3.574 & 3.690 \\
\hline$(4)$ & Voltage & $\mathrm{V}$ & 6100 & 6085 & 6050 & 6090 & 6086 \\
\hline$(5)$ & Current & $\mathrm{A}$ & 67.40 & 65.92 & 62.69 & 60.70 & 56.60 \\
\hline$(6)$ & The input power of electric machine & $\mathrm{kW}$ & 579.79 & 565.02 & 538.87 & 525.36 & 487.28 \\
\hline$(7)$ & The power of pump shaft & $\mathrm{kW}$ & 545.12 & 533.55 & 515.01 & 507.88 & 490.47 \\
\hline$(8)$ & The speed of pump shaft & $\mathrm{r} / \mathrm{min}$ & 1475 & 1479 & 1479 & 1470 & 1471 \\
\hline \multirow{3}{*}{ After conversion } & The rate of flow & $\mathrm{m}^{3} / \mathrm{h}$ & 447.83 & 435.89 & 405.27 & 383.93 & 351.20 \\
\hline & The head of delivery & $\mathrm{m}$ & 323.75 & 331.30 & 339.39 & 359.08 & 369.71 \\
\hline & The pump efficiency & $\%$ & 72.47 & 73.66 & 70.17 & 68.34 & 67.98 \\
\hline
\end{tabular}

TABLE 2: The test data through the ultrasonic flowmeter method.

\begin{tabular}{lccccccc}
\hline \multirow{2}{*}{ Number } & \multirow{2}{*}{ Item } & \multirow{2}{*}{ Unit } & \multicolumn{4}{c}{ The adjustment point of flow } \\
& & & 1 & 2 & 3 & 4 \\
\hline$(1)$ & The flow & $\mathrm{m}^{3} / \mathrm{h}$ & 449.03 & 437.19 & 407.55 & 388.02 \\
$(2)$ & The pump efficiency & $\%$ & 72.40 & 74.13 & 70.64 & 69.89 \\
\hline
\end{tabular}

\section{Measures for Ensuring the Testing Precision}

The accuracy of the measuring devices must be ensured in order to improve the testing precision of the pump efficiency. But the accuracies of the testing results are influenced inevitably by the on-site installation and operation and other human factors [14-16]. In order to further ensure the testing accuracies, it is better to adopt the following measures:

(1) There should not be other heat sources in the water flow at inlet and outlet of the pump.

(2) A buffer damping device should be provided under the pressure sensor to avoid the pressure fluctuating too greatly to collect the data accurately.

(3) Considering that the pressure at inlet is negative, a pressure sensor with proper range should be chosen; and the pressure sensors at inlet and outlet should not be exchanged during testing.

(4) The temperature sensors and the pressure sensors should be regulated to zero before testing and calibrated at regular intervals to improve the testing precisions and reduce the errors caused by measuring devices themselves.

(5) The following should be noted when testing temperatures: keep away from the unstable flow filed, keep far away from the sections of pipelines, which have rapid changes, and insert the probe of the temperature entirely into the protective sleeve in order to avoid the influence exerted by the flowing air of outside to it; make the probe of temperature cling to the inner wall of the sleeve, inject the water of the water sump and heat-conduction oil into the sleeve, and seal the head of the sleeve with rubber in order to prevent the water from flowing out.

(6) The protective sleeves of the temperature sensors should be machined based on the pipelines with different pipe diameters. It is better that the protective sleeves be inserted into the middle of the pipelines, too long or too short being not suitable.

(7) The pressure and temperature sensors at inlet and outlet should be close to the pump body as much as possible in order to ensure the accuracies of testing data.

\section{Conclusion}

(1) The thermodynamics calculation equations for testing the pump efficiency, which meet the requirements for the engineering on-site testing precisions, are derived from the enthalpy and entropy theories of water based on the law of conservation of energy and the law of conversion.

(2) The protective sleeves and the bases of the sensors for measuring the temperatures at inlet and outlet of the pump have been designed, which can help the sensors measure the temperatures of water at inlet and outlet of the pump accurately and conveniently. Thus, it is realized that the thermodynamics method can be used for testing the pump efficiency.

(3) It is proved that the thermodynamics method can meet the requirement for precision of engineering onsite testing in coal mine by on-site experiments. It is better to apply the thermodynamics method to the 
main drainage pump in coal mine with large flow and high head delivery. For the small-sized drainage pump at room temperature, the thermodynamics method is not applicable, because the data collected from the sections of the inlet and outlet of the pump lack uniformity, the measuring devices have limitations, and the correction values are faulty.

(4) The measures for testing the pump efficiency using the thermodynamics method have been proposed, which have directive significance to the on-site testing of the pump efficiency in coal mine.

\section{Competing Interests}

The author declares that there is no conflict of interests regarding the publication of this paper.

\section{Acknowledgments}

This research was supported by the National Natural Science Foundation of China (50775213) and the specialized research fund for the doctoral degree in colleges and universities funded projects (200502900).

\section{References}

[1] Z. Yu, X. Li, D. Shang, H. Wu, and J. Li, "Application of inverter in the coal mine," in Proceedings of the International Conference on Measuring Technology and Mechatronics Automation (ICMTMA '10), pp. 634-637, Changsha, China, March 2010.

[2] M. A. El-Naggar, "A one-dimensional flow analysis for the prediction of centrifugal pump performance characteristics," International Journal of Rotating Machinery, vol. 2013, Article ID 473512, 19 pages, 2013.

[3] H. Liu, S. Z. Chen, R. J. Chen et al., "Research on scaling mechanism of pump house in Hanqiao Coal Mine," Journal of Safety and Environment, vol. 5, no. 3, pp. 106-110, 2005 (Chinese).

[4] F. Papa and D. Radulj, "Canada takes a lead in benchmarking pump energy efficiency," Water Efficiency, pp. 30-33, 2013.

[5] A. C. Bwalya and M. W. Johnson, "Experimental measurements in a centrifugal pump impeller," Journal of Fluids Engineering, vol. 118, no. 4, pp. 692-697, 1996.

[6] F. Papa, D. Radulj, B. Karney, and M. Robertson, "Pump energy efficiency field testing and benchmarking in Canada," Journal of Water Supply Research and Technology AQUA, vol. 63, no. 7, pp. 570-577, 2014.

[7] J. Wang and C. X. Li, "A new pump efficiency mathematical model based on thermodynamic method and application," Journal of Nan Jing College of Engineering, vol. 8, no. 3, pp. 33-36, 2010.

[8] K. Ghorbanian and M. Karimi, "Thermodynamic analysis of a hybrid gas turbine/thermoacoustic heat pump/refrigeration engine," International Journal of Exergy, vol. 15, no. 2, pp. 152170, 2014.

[9] A. J. Milne, "Considerations when using the thermodynamic method of pump testing," World Pumps, vol. 7, pp. 23-26, 1990.

[10] A. J. A J Milne, "Implications of measurement uncertainty when using the thermodynamic method of pump efficiency monitoring in water," Proceedings of the Institution of Mechanical
Engineers, Part A: Journal of Power and Energy, vol. 204, no. 2, pp. 109-119, 1990.

[11] AQ 1012-2005 The Main Drainage System Safety Testing Inspection Specifications in Coal Mine, China Standards Press, Beijing, China, 2005.

[12] GB3216-89, The Centrifugal Pump, The Mixed Flow Pump, Axial Flow Pump and Vortex Pump Test Method, China Standards Press, Beijing, China, 1989.

[13] L. An, S. Wang, and P. Qin, "Applied research on measurement and on-line monitoring of pump efficiency and flow using thermodynamic method," Zhongguo Dianji Gongcheng Xuebao/Proceedings of the Chinese Society of Electrical Engineering, vol. 11, no. 2, pp. 36-40, 1994.

[14] X. Liu, B. Karney, R. Djordje, and Z. Wang, "Application of thermodynamic method in pump efficiency measurement, and comparison with conventional method," Journal of Mechanical Engineering, vol. 51, no. 10, pp. 189-196, 2015.

[15] Z. S. Qi, "Analysis of temperature of underground heat exchanger and efficiency of heat pump with combined cooling and heating," Journal of Jilin University, vol. 42, no. 2, pp. 339343, 2012.

[16] D. Radulj, F. Papa, and B. Karney, "Ontario wastewater pump efficiency testing program," in Proceedings of the Water Environment Association of Ontario Ottawa Technical Conference, Ottawa, Canada, 2012. 


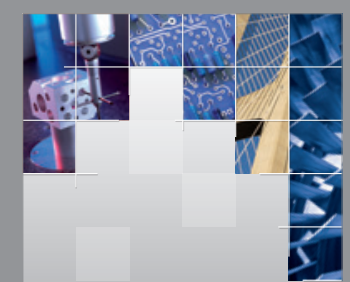

\section{Enfincering}
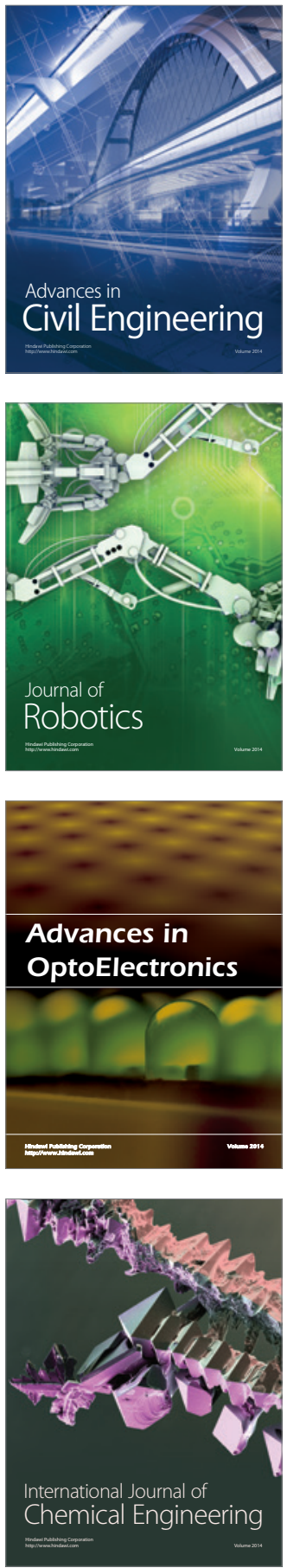

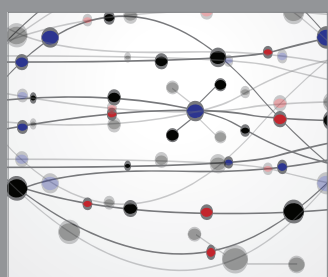

The Scientific World Journal

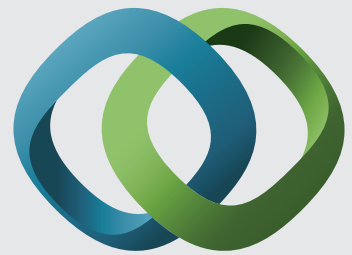

\section{Hindawi}

Submit your manuscripts at

https://www.hindawi.com
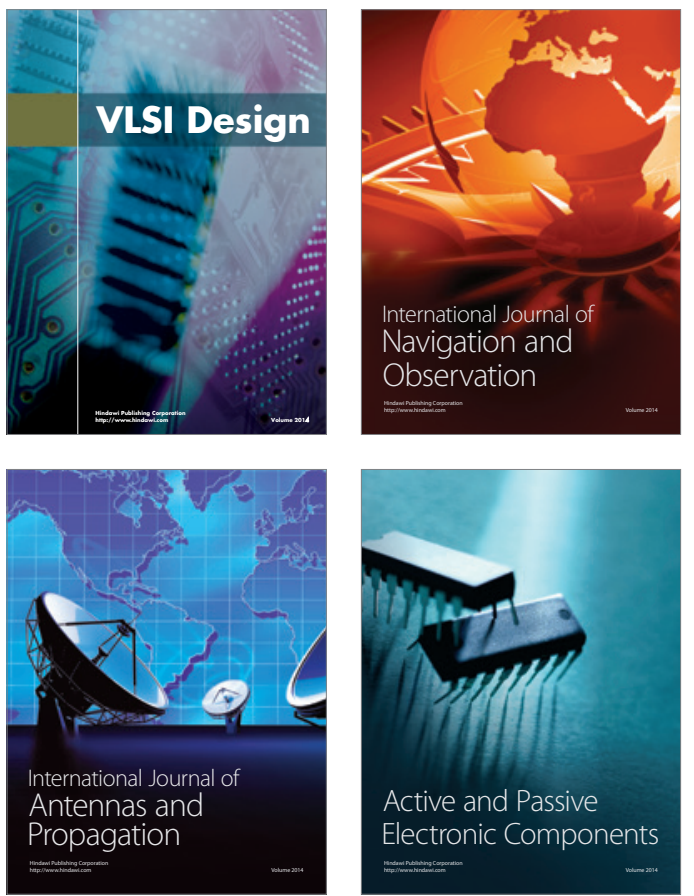
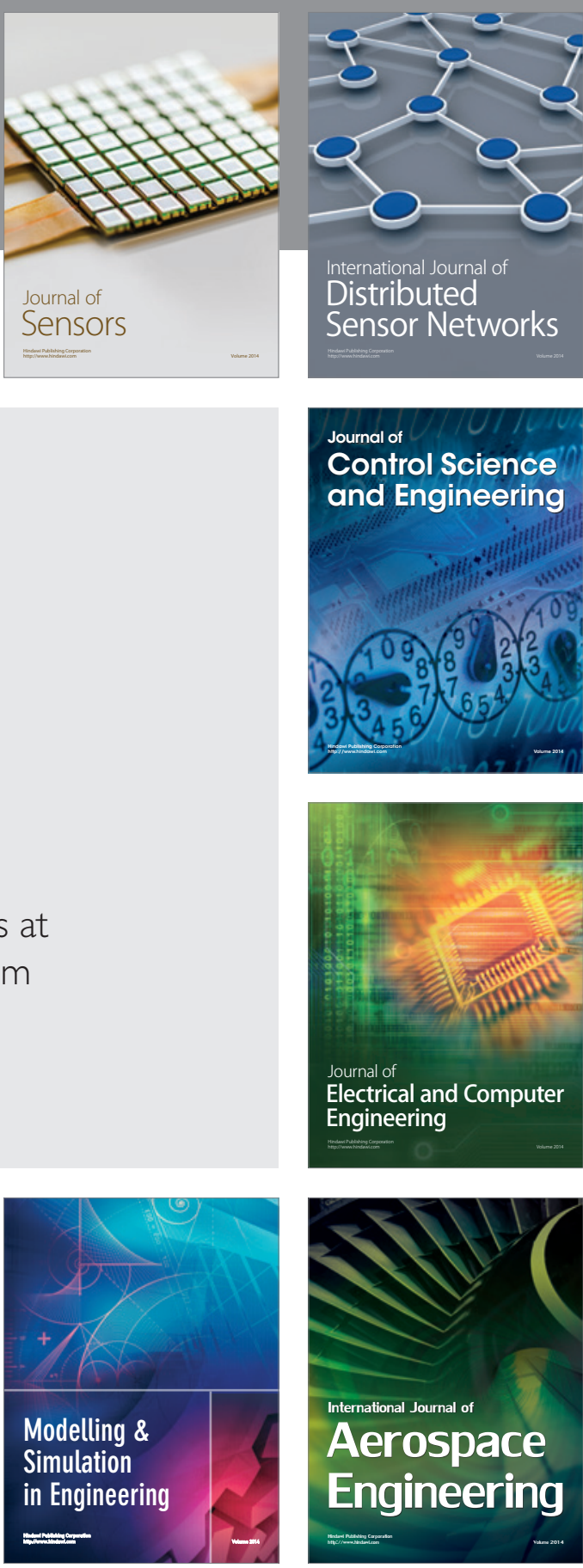

International Journal of

Distributed

Sensor Networks

$-$

Joumal of

Control Science

and Engineering
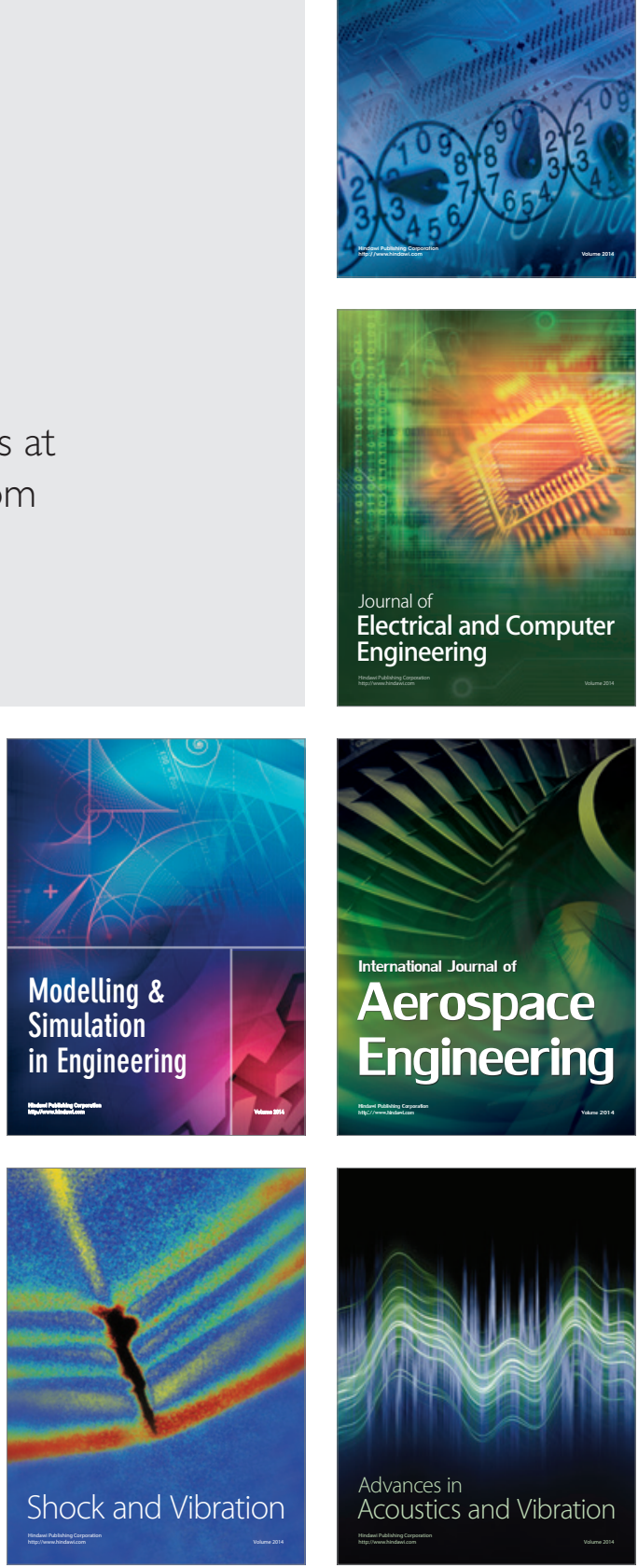